\title{
Potential Application for Micro-Particles Manipuation Utilizing Electrokinesis in an Electrodeless Dielectrophoresis Chip
}

\author{
Liang-Ju Chien, Chen- Yu Lee, Jia-Cheng Pan, Yao-Lin Gao, Chi-Han Chiou, and Jr-Lung Lin
}

\begin{abstract}
This study presented the feasibility of the microparticle manipulation, i.e., trapping and separation approach, utilizing an electrodeless dielectrophoresis microfluidic chip. Experimental observations were facilitated to investigate the microparticle electrokinesis behavior by tuning of the operation parameters, i.e., driving frequency and applied potential. These results not only revealed the fluid cloud be generated an electroosmotic flow (EOF), but also categorized the optimum trapping/separating schemes for the different particle sizes. Besides, the proposed operating mode can be used to continuously trap/separate particles and simultaneously convey them through the microfluidic device, eliminating the need for an additional micropump. Certainly, the device can be envisioned as a single automated device that supports the multi-functions such as transportation, separation, and detection, facilitating the realization of a Lab-on-a-Chip.
\end{abstract}

Index Terms-trapping, separation, electrodeless dielectrophoresis, electroosmosis, dielectrophoresis.

\section{INTRODUCTION}

In the past decades, cell or microparticle separation has gained significant attentions in sample preparations for biological and chemical applications especially in microfluidic systems. Several approaches were employed the application for particle manipulation, included optical [1], mechanical [2], magnetic [3], dielectrical [4], and other manipulations [5]. For examples, flow cytometry was used for cell sorting and characterization [6], laser tweezers for cell manipulation [7], and antibody-labeled magnetic beads for cell separation. Among these methods, optical manipulation is known as the non-contact and contamination-free manipulation, but the complicated optical setup, complex operation, and expensive instrumentation to limit their further applications in microfluidics.

Currently, an alternative separation method is

Manuscript received April 24, 2012; revised May 30, 2012.This work was supported by the National Science Council in Taiwan (NSC100-2221-E-214-012)

J.-L. Lin is with Department of Mechanical and Automation Engineering, I-Shou University, Kaohsiung, Taiwan (tel.: +886-7-6577711 Ext3320; e-mail: lj1@isu.edu.tw.).

L.-J. Chien and C.-H. Chiou both served as a principal investigator in Microsystems Technology Center at ITRI South, Industrial Technology Research Institute, Taiwan (e-mail: liangju95@gmail.com, prayjohn@itri.org.tw )

C.-Y. Lee, J.-C. Pan, and Y.-L. Gao all are in Department of Mechanical and Automation Engineering, I-Shou University, Kaohsiung, Taiwan (e-mail: sken662002@yahoo.com.tw, william@major.com.tw and isu9904005m@isu.edu.tw). dielectrophoresis (DEP), which be briefly cataloged two types, namely electrode-arrayed DEP [8] or electrodeless DEP (EDEP) [9]. Electrode-arrayed DEP exhibits many advantages such as flexibility, controllability and ease of application. Also, it has been proven to be an efficient non-invasive method for separating various cell types without any need for labeling. However, electrode-arrayed DEP causes fast decay of the field gradient to damage the bio-sample. These results significantly yield to reduce the trapping efficiency and throughput. Therefore, a concept of insulator-based (electrodeless) DEP (iDEP or EDEP) was introduced to avoid some of the aforementioned problems that are associated with the use of electrode-arrayed electrodes. EDEP can be performed using different geometries and configurations of insulating structures, of course, the triangular constricted structure is the most attractive because the field gradient can be preserved over the entire cross section of the trap. Under a highly non-uniform electric field caused by a constricted gap, polarizable particles experience a force in the direction along (positive DEP, pDEP) or against (negative DEP, nDEP) the electric field gradient depending on their dielectric properties. However, by applying an electrostatic field to a conductive fluid, the phenomena of EOF and DEP innately coexists in a world-nano-micro environment. The magnitudes of influence of EOF and DEP on bioparticles are determined on many physical and chemical properties, i. e., solid surface, particles, medium, and even the geometries of insulator [9]-[11]. Based on these varieties of properties, many derivative applications have been reported for particle manipulation and liquid conveyance using DEP or EOF. Typically, EOF drag force is mainly used to both convey particles and liquids [8], [12]. Recently, biomolecules can be continuously trapped and collected in world-nano-micro interfacing by utilizing EOF transport mechanism [13], ]14]. On the other hand, DEP force has demonstrated for trapping, fusing, sorting, and lysing biological materials [15], [16]. These exhibited the versatile capabilities of the techniques by a means of electrokinesis based on varied physical mechanisms.

In this study, an EDEP microfluidic chip was successfully demonstrated to achieve the microparticle manipulation, i.e., trapping or separation. The different sizes of microparticles can be easily trapped at the different locations of the EDEP microfluidic chip by tuning of the applied voltages. Experiments revealed that microparticle separation is significantly dominated by tuning of the different applied potentials. It can be used to continuously separate the microparticles without an external transportation system. 


\section{THEORY}

\section{A. Dielectrophoresis}

An assuming spherical particle was sounded in a conductive medium, the DEP force exerted on a particle was expressed [17] by the followings form:

$$
F_{D E P}=2 \pi a^{3} \varepsilon_{m} \operatorname{Re}[C M] \nabla E^{2}
$$

where $a$ is the radius of the particle; $\varepsilon_{\mathrm{m}}$ is the permittivity of the medium; $E$ is the amplitude of the electric field. $\operatorname{Re}[C M]$ is the real part of the complex Clausius-Mossotti factor. Basically, DEP force, $F_{D E P}$, is proportional to the gradient of the square of the applied electric field and to the third power of the particle radius. Here, the complex Clausius-Mossotti factor (CM) was obtained by

$$
\begin{aligned}
& C M=\frac{\varepsilon_{p}^{*}-\varepsilon_{m}^{*}}{\varepsilon_{p}^{*}+2 \varepsilon_{m}^{*}} \\
& \varepsilon^{*}=\varepsilon-j \frac{\sigma}{w}
\end{aligned}
$$

Here $\varepsilon^{*}$ is the complex permittivity; $\sigma$ is conductivity; $w$ is the angular frequency of the electric field; and $j$ is the imaginary unit. The subscripts $p$ and $m$ refer to the particle and the medium, respectively. In principle, the real part of $\operatorname{Re}[C M]$ is bounded between 1.0 and -0.5 .

\section{B. Electroosmosis}

Electroosmotic flow (EOF) is caused by the force exerted on the EDL by an applied tangential electric field. In planar microelectrode arrays are used to induce direct current (DC) electrokinetics, divergent electric fields are generated such that a component of the electric field lies tangential to the EDL, which is induced on the electrode surface. Accordingly, ions inside the diffuse double layer experience a force that, on average over time, acts from the edge, across the surface of the electrode. In this study, EOF velocity $\left(u_{E O F}\right)$ was expressed by

$$
u_{E O F}=\frac{-\varepsilon_{m} \zeta E_{x}}{\eta}
$$

where $\eta$ is medium viscosity; $\zeta$ is zeta potential of microchannel wall; $E_{x}$ is the x-directional electric field strength and is also strong functions of the applied voltage as well as the driving frequency. Assuming that spherical particles travel into the viscous medium, the Stoke drag force is given by:

$$
F_{s t}=6 \pi \eta a\left(u_{E O F}-u_{p}\right)
$$

where $u_{E O F}$ and $u_{p}$ denote the velocities of the medium and the microparticle. The Stokes force is linearly proportional to the velocity and the particle radius, respectively.

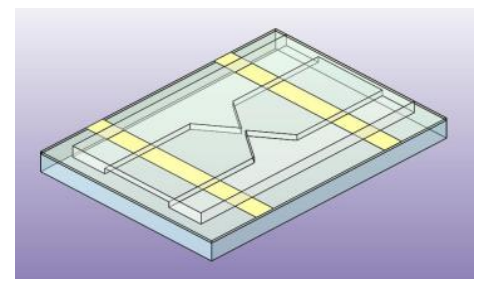

Fig. 1. Schematic EDEP microfluidic device

\section{MATERIAL AND METHODS}

\section{A. Design and Fabrication}

The proposed EDEP microfluidic device, comprising a pair of triangular insulating structures, a microfluidic channel, and a pair of driving electrodes, schematically depicts as shown as Figure 1. Two $60^{\circ}$-triangular insulating structures were constructed a constricting gap of $5 \mu \mathrm{m}$ to squeeze the electric field in a conductive solution to produce a highly electric field gradient for trapping particles. A pair of driving electrodes separated by $2 \mathrm{~mm}$ was adopted to produce the possible required electric field in the microchannel. The microchannel dimension exhibits $1000 \mu \mathrm{m}$ width and $5 \mu \mathrm{m}$ depth, respectively.

The EDEP microfluidic chip was fabricated using Micro-electromechanical Systems (MEMS) technology as

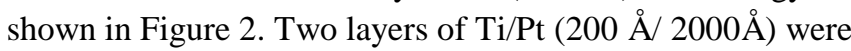
deposited on a glass substrate to form a pair of electrodes by using the lift-off process (Figs. 2(a) and (b)). A silicon wafer was patterned using standard photolithography, and then reactive ion etching (RIE) was employed to produce a $5 \mu \mathrm{m}$ deep mold with a pair of triangular insulating structures and the microfluidic channel (Fig. 2(c)). Accordingly, the inverse structures with patterned features were cast using PDMS materials and the Si mold (Fig. 2(d)). Finally, the replicated PDMS structures (Fig. 2(e)) and the glass substrate with $\mathrm{Pt}$ electrodes were bonded each other by oxygen plasma treatment to yield a complete EDEP microfluidic chip (Fig. 2(f)). Figure 3(a) shows the assembly of the EDEP chip. Figure 3(b) displays the scanning electron microscope (SEM) image of the mold.

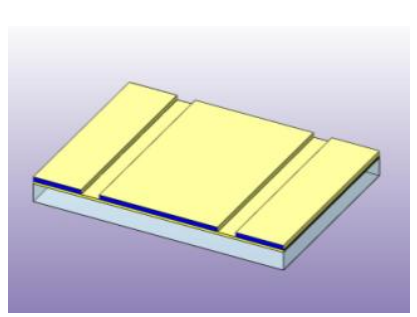

(a)

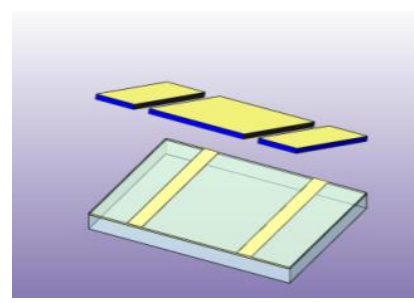

(b)

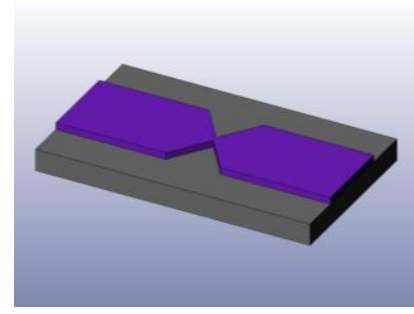

(c)

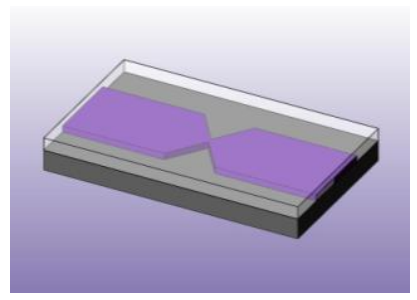

(d)

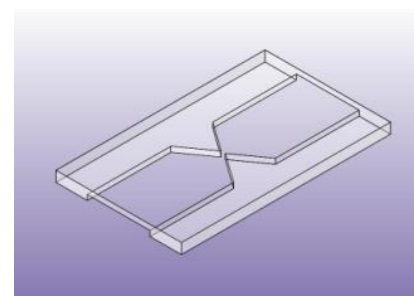

(e)

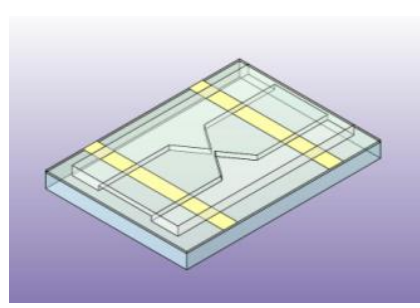

(f)
Fig. 2. Simplified fabrication process of EDEP microfluidic chip. 


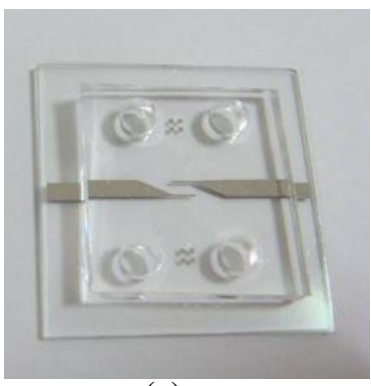

(a)

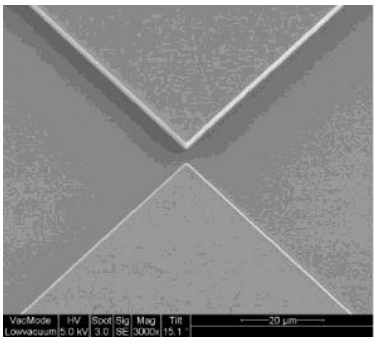

(b)
Fig. 3. (a) Photograph of assembly of EDEP microfluidic chip. (b) SEM image of a pair of triangular insulating structures.

\section{B. Experiment}

Experimental setup involved a signal generator (33220A, Agilent, USA), an oscilloscope (TDS 1002B, Tektronix, USA) and a power amplifier (LPA 400, Newtons4th, UK) was employed for the investigation of EDEP microfluidic chip. The EDEP microfluidic chip was mounted on top of an inverted microscope (DMI 4000B, Leica, Germany) for microparticle visualization. Three polystyrene microparticles (excitation $580 \mathrm{~nm} / \mathrm{emission} 605 \mathrm{~nm}$, Invitrogen, USA) of $0.5-, 1.0-$, and $2.0-\mu \mathrm{m}$ were used to experimentally investigate the electrokinesis effect. The fluorescence images of the microparticles were captured using a $10 \mathrm{X}$ or $20 \mathrm{X}$ objective len, as well as a cool CCD (Charge-coupled Device) (Cool SNAP HQ ${ }^{2}$, Photometrics, USA). Besides, the viscosity of solution was adjusted to be $1 \mathrm{cP}$ and a buffer solution of 10 $\mathrm{mM}$ Tris- $\mathrm{HCl}(\mathrm{pH} 8.0)$ with a fixed conductivity of 730 $\mu \mathrm{S} / \mathrm{cm}$. All experiments were conducted in a range of electric field $40 \sim 600 \mathrm{~V}_{\mathrm{pp}} / \mathrm{cm}$ with a fixed driving frequencies $7 \mathrm{kHz}$ or $10 \mathrm{kHz}$.

\section{RESULTS AND DISCUSSION}

\section{A. EOF Characterization on Frequency Effect}

Theoretically, two forces exerted on the trapped particles were taken into a consideration of electrokinesis effect. The first one is the DEP force $\left(F_{D E P}\right)$, which acts as a holding force to stop the particles from moving. The other one is Stoke's force $(F s t)$, which EOF acts on the particles to transport. Therefore, the net motion of particles is determine by both EOF and DEP effect. Figure 4(a) reveals that the fluorescent $0.2-\mu \mathrm{m}$ particles passed through the constriction when a field with a frequency from $100 \mathrm{~Hz}$ to $5 \mathrm{MHz}$ was continuously applied. The microparticles flowed forward through the constricting gap by EOF conveyance as the driving frequency is below $810 \mathrm{kHz}$. Conversely, the flow direction reversed, i.e., backward flow, as the driving frequency is beyond 810 kHz. Experimental observation exhibited EOF effect was occurred within a pair of triangular insulating constructions. It also showed the higher velocities occurred near the constriction wall due to the zeta potential effect. Interestingly, a reciprocating motion was experimentally observed at a frequency of $810 \mathrm{kHz}$. The relationship of the EOF velocity and the driving frequencies as plotted in Fig. 4(b). It is clearly seen that the EOF velocity initially increases with the operation frequency, and then EOF velocity reaches a maximum value and subsequently declines to a negative EOF velocity as the operation frequency is increased further.
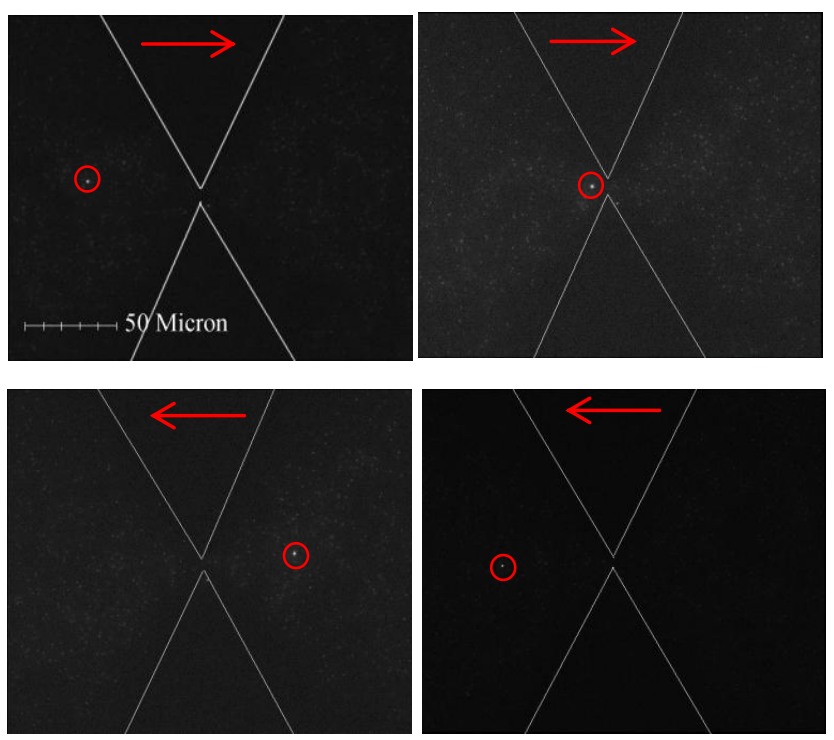

(a)

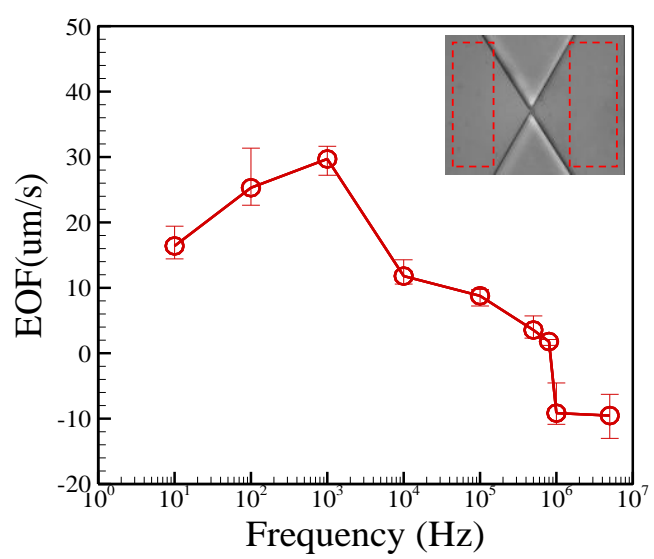

(b)

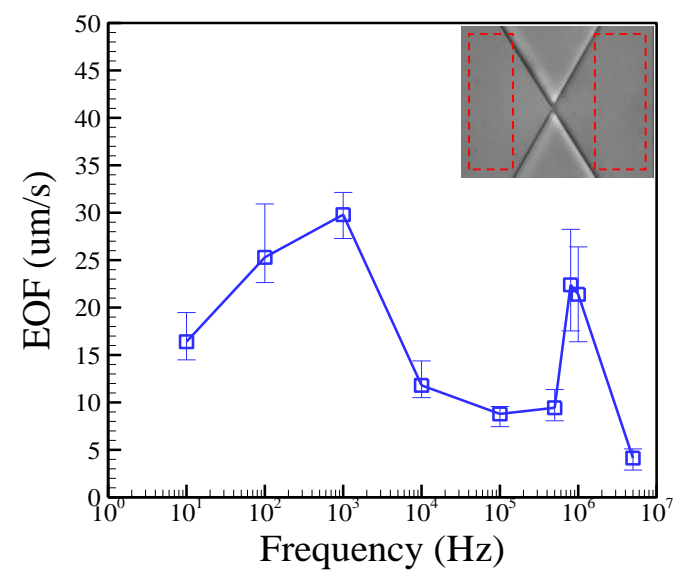

(c)

Fig. 4. (a) Conveyance of $0.2 \mu \mathrm{m}$ particles under electroosmosis. The red arrow indicated the flow direction. Relationship between EOF velocities against different frequencies for (b) continuously and (c)non-continuously operation modes. Notably, two red dash-line regions indict to experimentally calculate mean EOF velocity. The applied voltage was kept at a constant of $90 \mathrm{~V}_{\mathrm{pp}} / \mathrm{cm}$

Notably, the driving frequencies were not continuously applied on to the EDEP chip, the flow filed generated a forward flow but the fluid backflow not to occur. Fig. 4(c) plotted the relationship of the EOF velocity and the different driving frequencies. The EOF profile is a similar pattern with 
the continuously operation (referred to Fig. 4(b)) as the driving frequency is lower than $10 \mathrm{kHz}$. Conversely, the flow behavior is significantly different with Fig. 4(b) as the driving frequency is over $10 \mathrm{kHz}$. EOF velocities slightly decreased with the increasing frequencies and the suddenly go up and go down with the increasing frequencies. But, the phenomenon of fluid backflow didn't be taken place at the higher driving frequency. Here exhibits two peaks velocities at the frequency of $1.0 \mathrm{kHz}$ and $1.0 \mathrm{MHz}$, respectively.

\section{B. DEP Characterization on Potential Effect}

1.0-um particles were manipulated at the different potential ranged of $100 \sim 500 \mathrm{Vpp} / \mathrm{cm}$. A potential of $100 \mathrm{Vpp} / \mathrm{cm}$ was applied on the EDEP chip, then 1.0-um particles were trapped closed to the gap of the constriction (Fig. 5(a)). When the potential gradually increased up to $500 \mathrm{Vpp} / \mathrm{cm}, 1.0-\mathrm{um}$ particles were travelled away from the gap of the constriction by the negative DEF force(Figs. 5(b), 5(c) and 5(d)). Conversely, when the potential generally decreased down to $100 \mathrm{Vpp} / \mathrm{cm}, 1.0$-um particles were pulled close toward the gap of the constriction (Figs. 5(e) and 5(f)). The similar manners were also taken place for those particles of 0.5 - and 2.0- $\mu \mathrm{m}$ particles.

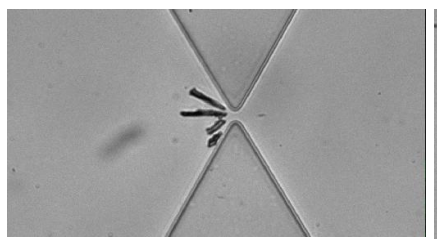

(a)

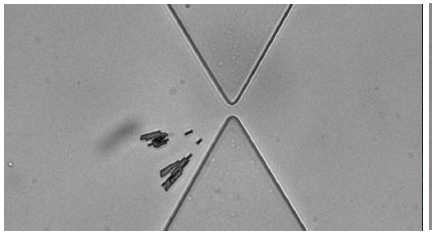

(c)

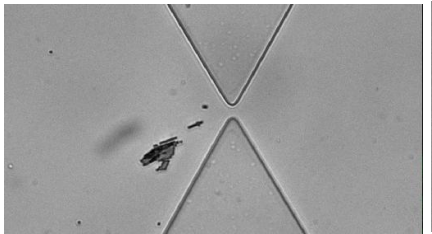

(e)

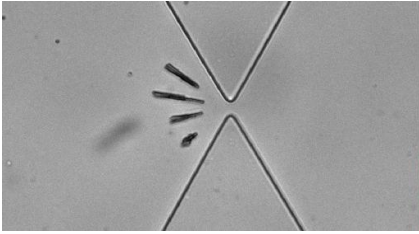

(b)

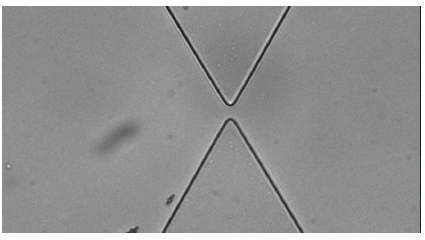

(d)

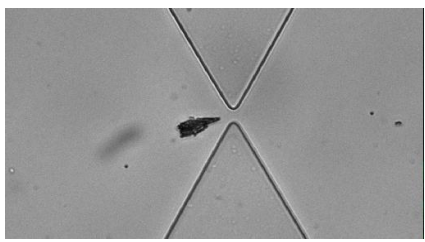

(f)
Fig. 5. A series of images for the 1.0-um particles manipulation at the different potentials of (a)100; (b)200; (c)400; (d)500; (e)300 ; (f) 100 $\mathrm{Vpp} / \mathrm{cm}$ with a constant frequency of $7 \mathrm{kHz}$.

Next, 0.5-, 1.0- and 2.0- $\mu \mathrm{m}$ particles are independently operated to evaluate the trapping effect in the proposed EDEP microfluidic chip at a range of applied electric field 40 600 $\mathrm{Vpp} / \mathrm{cm}$ with a fixed frequency $10 \mathrm{kHz}$. Table 1 showed the different microparticle sizes whether trapped or not. " $\bigcirc$ " indicted the microparitlces can be trapped and " $x$ " presented that cannot be trapped. It was clearly seen that $0.5-\mu \mathrm{m}$ particles can be trapped for the potential of 100 500 Vpp/cm, $1.0-\mu \mathrm{m}$ particles for that of $80 \sim 500 \mathrm{Vpp} / \mathrm{cm}$, and $2.0-\mu \mathrm{m}$ particles for that of $50 \sim 500 \mathrm{Vpp} / \mathrm{cm}$. It was facilitated to easily separate for the $0.5-, 1.0-$ and $2.0-\mu \mathrm{m}$ particles by adjusting the driving potential.

TABLE I: DRIVING POTENTIALS AND MiCROPARTICEL SIZES FOR THE TRAPPING EFFECT

\begin{tabular}{|c|c|c|c|}
\hline Size $(\mu \mathrm{m})$ & 0.5 & 1.0 & 2.0 \\
\hline Potential(Vpp/cm) & & & \\
\hline 40 & $\times$ & $\times$ & $\times$ \\
\hline 50 & $\times$ & $\times$ & $\bigcirc$ \\
\hline 60 & $\times$ & $\times$ & $\bigcirc$ \\
\hline 70 & $\times$ & $\times$ & $\bigcirc$ \\
\hline 80 & $\times$ & $\bigcirc$ & $\bigcirc$ \\
\hline 90 & $\times$ & $\bigcirc$ & $\bigcirc$ \\
\hline 100 & $\bigcirc$ & $\bigcirc$ & $\bigcirc$ \\
\hline 200 & $\bigcirc$ & $\bigcirc$ & $\bigcirc$ \\
\hline 300 & $\bigcirc$ & $\bigcirc$ & $\bigcirc$ \\
\hline 400 & $\bigcirc$ & $\bigcirc$ & $\bigcirc$ \\
\hline 500 & $\bigcirc$ & $\bigcirc$ & $\bigcirc$ \\
\hline 600 & $\times$ & $\times$ & $\times$ \\
\hline
\end{tabular}

\section{Particle Separation Characterization}

Since the DEP force is proportional to the cube of the cell diameter, even slight size variation cannot result in significant differences in DEP force. Therefore, DEP separation based solely on cells' dielectric properties is difficult for separating two or more populations of cells with similar sizes. Figure 6 shows the different size of microparticle individually trapped in the EDEP chip. The operation potential and frequency are $500 \mathrm{Vpp} / \mathrm{cm}$ and $10 \mathrm{kHz}$, respectively. Clearly, the n-DEP force acted locally on the microparticles trapped in the constriction region. Basically, the DEP force acted locally on the small size particles trapped close to the constriction. Conversely, the larger size particles are trapped farer from the gap. Notably, the microparticles were trapped dielectrophoretically at one side of the gap and the particle-trapped contours demonstrated the semicircle shape. The results also reveal that the trapped distance increases with the increasing operating potential.

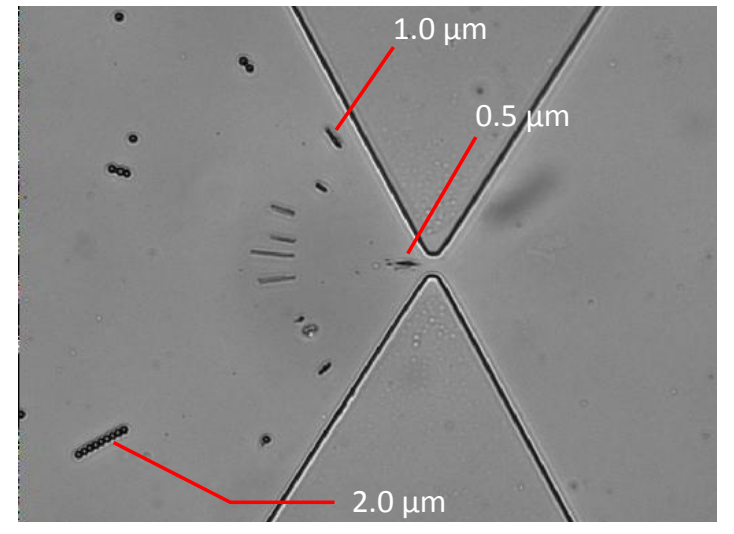

Fig. 6. Individually trapped image of $0.5-, 1.0-$ and $2.0-\mu \mathrm{m}$ particles at the driving potential and frequency are $500 \mathrm{Vpp} / \mathrm{cm}$ and $10 \mathrm{kHz}$. (b) Trapped distance against different particle size at the range of potential 100 500 $\mathrm{Vpp} / \mathrm{cm}$ with a fixed frequency $10 \mathrm{kHz}$.

Finally, a solution mixed $0.5-, 1.0-$, and $2.0-\mu \mathrm{m}$ particles 
were pipetted into the reservoir, a driving electric potential with a fixed frequency $10.0 \mathrm{kHz}$ was continuously applied on the electrodes form 100 down to $40 \mathrm{Vpp} / \mathrm{cm}$. The micorparticles were continuously flowed by the presence of the EOF effect then were individually trapped in the DEP-affected area by the presence of the DEP effect, which was close to the constricting gap as shown in Fig. 7(a). Then, the operation potential was down to $90 \mathrm{Vpp} / \mathrm{cm}, 70 \mathrm{Vpp} / \mathrm{cm}$, and $40 \mathrm{Vpp} / \mathrm{cm}$, respectively, $0.5-\mu \mathrm{m}, 1.0-\mu \mathrm{m}$ and $2.0-\mu \mathrm{m}$ particles were were passed indivually through the gap of the constriction (Figs. 7(b), 7(d) and 7(f)). Hence, we have sucessfully demonstrated an approach to continousely separate the diferent sizes of microparticles by tuning of driving potential.

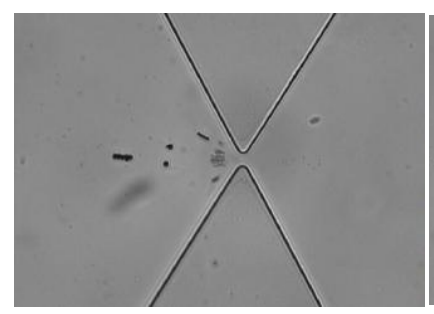

(a)

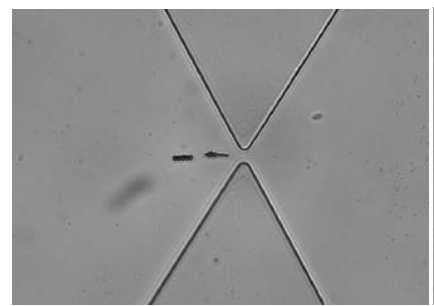

(c)

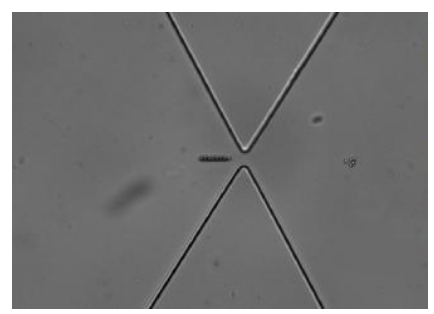

(e)

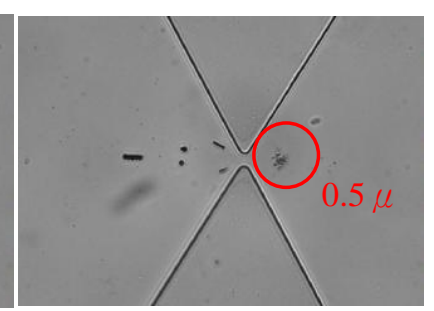

(b)

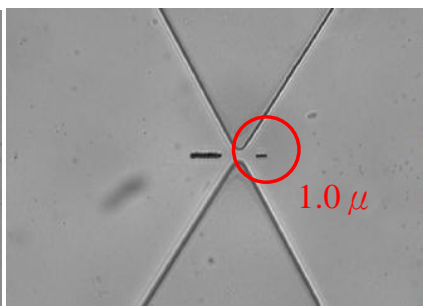

(d)

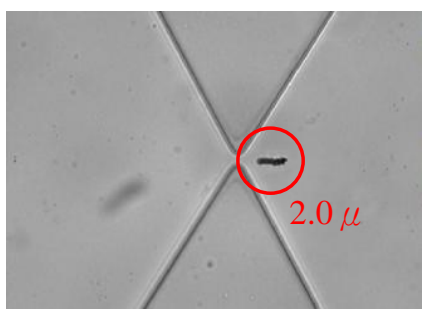

(f)
Fig. 7. A series of images for the continuously separation of $0.5-, 1.0$ - and $2.0-\mu \mathrm{m}$ particles at the driving electrical potential of (a) $100 \mathrm{Vpp} / \mathrm{cm}$; (b) 90 $\mathrm{Vpp} / \mathrm{cm}$; (c) $80 \mathrm{Vpp} / \mathrm{cm}$; (d) $70 \mathrm{Vpp} / \mathrm{cm}$; (e)50 Vpp/cm; (f) $40 \mathrm{Vpp} / \mathrm{cm}$ with a given frequency of $10.0 \mathrm{kHz}$.

\section{CONCLUSION}

This work have sucessfully demonstrated the feasibility of the microparticle trapping and separation approach utilizing an EDEP microfluidic chip. The tarpping or separating effect dominates on whether EOF or DEP depended on the operation parameters, i.e., applied potential and driving frequency. Experimetally observed that $0.5-\mu \mathrm{m}$ particles were trapped for the potential of 100 500 Vpp/cm, $1.0-\mu \mathrm{m}$ particles for that of $80 \sim 500 \mathrm{Vpp} / \mathrm{cm}$, and 2.0- $\mu \mathrm{m}$ particles for that of 50 500 Vpp/cm. Furthermore, this apporach can be used to continousely flow for the separatation of the microparictles.

\section{REFERENCES}

[1] S. L. Friedman and F. J. Roll, "Isolation and culture of hepatic lipocytes, Kupffer cells, and sinusoidal endothelial cells by density gradient centrifugation with," Stractan Anal.Biochem., vol. 161, pp. 207-218, 1987.

[2] L. Zhu, Q. Zhang, H. H. Feng, S. Ang, F. S. Chau, and W.T. Liu, "Filter-based microfluidic device as a platform for immunofluorescentassayofmicrobial cells," Lab on a Chip, vol. 4, pp. 337-341, 2004.

[3] M. Berger, J. Castelino, R. Huang, M. Shah, and R. H. Austin, "Design of a microfabricated magnetic cell separator," Electrophoresis, vol. 22, pp.3883-3892, 2001.

[4] M. Durr, J. Kentsch, T. Muller, T. Schnelle, and M. Stelzle," Microdevices for manipulation and accumulation of micro- and nanoparticles by dielectrophoresis," Electrophoresis, vol. 24, pp. 722-731, 2003.

[5] A. R. Minerick, R. Zhou, P. Takhistov, and H.C. Chang, "Manipulation and characterization of red blood cells with alternating current fields in microdevices," Electrophoresis, vol. 24, pp. 3703-3717, 2003.

[6] H. M. Shapiro, Practical flow cytometry, 1988, New York: AlanR.Liss.

[7] S. Chu, "Laser manipulation of atoms and particles," Science, vol. 253, pp. 861-866, 1991.

[8] E. B. Cummings, "Streaming dielectrophoresis for continuous-flow microfluidic devices," IEEE Eng. Med. Biol. Mag., vol. 23, pp.75-84, 2003.

[9] C. F. Chou and F. Zenhausern, "Electrodeless dielectrophoresis for micro total analysis systems," IEEE Eng. Med. Biol. Mag. vol. 22, pp.62-67, 2003.

[10] S. Ozuna-Chacón, B. H. Lapizco-Encinas, M. Rito-Palomares, S. O. Martínez-Chapa, and C. Reyes-Betanzo, "Performance characterization of an insulator-based dielectrophoretic microdevice," Electrophoresis, vol. 23, pp. 3115-3122, 2008.

[11] R. Yokokawa, Y. Manta, M. Namura, Y. Takizawa, N. C. H. Le, and S. Sugiyama, "Individual evaluation of DEP, EP and AC-EOF effects on DNA molecules in a DNA concentrator," Sens Actuators B: Chem., vol. 143, pp. 769-775, 2010.

[12] J. A. Oakley, K. J. Shaw, P. T. Docker, C. E. Dyer, J. Greenman, G. M. Greenway, and S. J. Haswell, "Development of a bi-functional silica monolith for electro-osmotic pumping and DNA clean-up/extraction using gel-supported reagents in a microfluidic device," Lab Chip, vol. 9, pp. 1596-1600, 2009.

[13] S. M. Kim, M. A. Burns, and E. F. Hasselbrink, "Electrokinetic protein preconcentration using a simple glass-poly(dimethylsiloxane) microfluidic chip," Anal Chem., vol. 78, pp. 4779-4785, 2006.

[14] J. H. Lee, Y. S. Song, and J. Han, "Multiplexed proteomic sample preconcentration device using surface-patterned ion-selective membrane," Lab Chip., vol. 8, pp. 596-601, 2008.

[15] R. Pethig and G. H. Markx, "Applications of dielectrophoresis in biotechnology, “ Trends Biotechnol., vol. 15, pp. 426-432, 1997.

[16] B. H. Lapizco-Encinas, B. A. Simmons, E. B. Cummings, and Y. Fintschenko, "Dielectrophoretic concentration and separation of live and dead bacteria in an array of insulators," Anal. Chem., vol. 76, pp.1571-1579, 2004.

[17] H. A. Pohl, "Dielectrophoresis: The behavior of neutral matter in nonuniform electricfelds," Cam bridge Cambridge UK, 1978, Chapter 4. 